\title{
A review of different resuscitation platforms during delayed cord clamping
}

\author{
Anup Katheria $\mathbb{1}^{1} \cdot$ Henry C. Lee $\mathbb{D}^{2} \cdot$ Ronny $\mathrm{Knol}^{3} \cdot$ Leigh Irvine ${ }^{4} \cdot$ Sumesh Thomas ${ }^{4}$
}

Received: 11 December 2020 / Revised: 10 March 2021 / Accepted: 29 March 2021 / Published online: 13 April 2021

(c) The Author(s), under exclusive licence to Springer Nature America, Inc. 2021

\begin{abstract}
There is a large body of evidence demonstrating that delaying clamping of the umbilical cord provides benefits for term and preterm infants. These benefits include reductions in mortality in preterm infants and improved developmental scores at 4 years of age in term infants. However, non-breathing or non-vigorous infants at birth are excluded due to the perceived need for immediate resuscitation. Recent studies have demonstrated early physiological benefits in both human and animal models if resuscitation is performed with an intact cord, but this is still an active area of research. Given the large number of ongoing and planned trials, we have brought together an international group that have been intimately involved in the development or use of resuscitation equipment designed to be used while the cord is still intact. In this review, we will present the benefits and limitations of devices that have been developed or are in use. Published trials or ongoing studies using their respective devices will also be reviewed.
\end{abstract}

\section{Introduction}

Globally, up to $10 \%$ of newborns require interventions to facilitate transition to extrauterine life while $1 \%$ need a more extensive resuscitative effort for stabilization [1]. The majority of infants undergo simple airway management, thermal care, and drying and stimulation to breathe. Others warrant extensive resuscitation and monitoring can include the application of a pulse oximeter and interventions such as positive pressure ventilation, chest compressions, endotracheal intubation, and drug administration [2]. Delayed cord clamping (DCC) has been shown to improve long-term neurological outcomes at 4 years of age in term infants, improve cognitive scores at 18 months, and reduce

Anup Katheria

anup.katheria@sharp.com

1 Neonatal Research Institute, Sharp Mary Birch Hospital, San Diego, CA, USA

2 Department of Pediatrics, Stanford University School of Medicine, Stanford, CA, USA

3 Department of Pediatrics, Erasmus Medical Centre-Sophia Children's Hospital, Rotterdam, The Netherlands

4 Foothills Medical Centre, Department of Pediatrics, University of Calgary, Calgary, AB, Canada mortality in preterm infants [3, 4]. While infants who need extensive resuscitation have higher morbidity [5], the majority of recommendations exclude these infants from receiving DCC, due to the need to move the infant to a resuscitation warmer that allows providers for adequate spacing and monitoring.

Infants who initiated spontaneous respirations had less blood retained in the placenta and higher hematocrits [6, 7]. Preterm infants require more time to establish breathing than their term counterparts, placing greater emphasis on the determination of breathing before the cord is clamped $[8,9]$. One epidemiologic study suggested that term newborns that take longer to breathe after their cord is clamped have a higher rate of admission to the NICU and increased mortality [10]. Another observational study of preterm infants demonstrated higher rates of intraventricular hemorrhage (IVH) and bronchopulmonary dysplasia in infants that were not breathing during DCC [11]. A study in anesthetized preterm lambs demonstrated positive pressure ventilation during DCC eliminated fluctuations in cardiac output and cerebral blood flow compared to early cord clamping [12]. Knol et al. have used the term physiological-based cord clamping (PBCC), where clamping of the cord only occurs when the infant is considered stable with a heart rate $>100$, arterial oxygen saturations above $90 \%$ and an oxygen requirement of $<0.4$.

Recent studies have demonstrated transitional improvements and possible neurodevelopmental benefits but this is 
Fig. 1 Life Start trolley. Labeled diagram for setup of preterm infant (left panel) and sketch of trolley during vaginal birth (right panel).

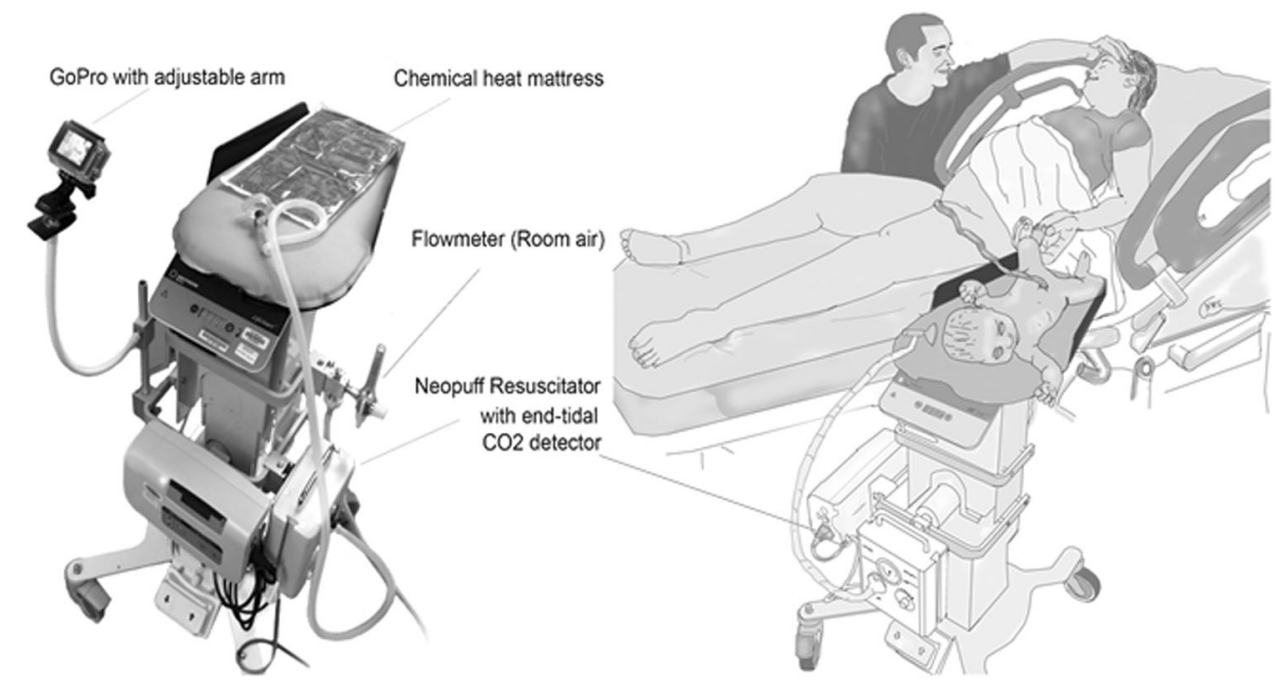

still an active area of research. We have brought together a group intimately involved in the development of resuscitation equipment used while the cord is still intact. In this review, we will present the benefits and limitations of devices that we developed or have been actively using. We will review completed or ongoing studies conducted using these resuscitation platforms.

\section{Lifestart ${ }^{\mathrm{TM}}$ Bed (Inspiration HealthCare group PLC, UK)}

One of the earliest commercially available devices was the LifeStart ${ }^{\mathrm{TM}}$ Trolley (FDA Class 1, CE certified) developed in the UK by a group of innovative engineer and obstetricians [13]. This device provides heat, allows for mounting of resuscitation equipment such as an oxygen blender with gas flow inlets, a ventilator, and has the ability to raise and lower using a foot pedal. The initial version had a shorter platform and had limited mobility to raise and lower. With the input of investigators, a second version of the device was developed, with a longer table and increased the range of the bed to move both higher and lower depending on the position of the mother.

\section{Clinical studies to date}

The LifeStart Trolley was first used as part of a large multicenter randomized controlled trial in the United Kingdom. One hundred and thirty-seven preterm infants randomized to resuscitation with an intact cord and 139 were clamped by $20 \mathrm{~s}$ of life. There was a suggestion of decreased mortality with resuscitation with an intact cord but it did not reach significance [14]. They also reported no difference in compliance between sites that used traditional resuscitation equipment and trolley. In the United States,
Katheria et al. began using the device as part of a pilot study to determine whether the provision of positive airway pressure during DCC in preterm infants had an improvement in placental transfusion compared to not providing DCC (Fig. 1, panel A). While they did not see any differences, the study was informative in that $90 \%$ of infants in both arms began breathing by $60 \mathrm{~s}$ of life [15]. In addition, the trial highlighted the challenges of using the trolley bed, which included difficulty of getting the trolley close enough to the infant often resulting in excessive traction on the cord or in some cases aborting the procedure and clamping the cord immediately.

A second study conducted by Katheria et al. was in term newborns $(N=60)$ that were at risk of needing resuscitation (Fig. 1, panel B) [16]. The study demonstrated physiological benefits with extended DCC (5 min with resuscitation if needed) compared to early cord clamping (1 min or less), but no clinical differences. This study highlighted the importance of oximetery, and the fact that it has been difficult to acquire such data during DCC. Quality improvement and feasibility projects have improved the compliance of using the LifeStart Trolley [17-19]. Further technology improvements will allow faster acquisition by oximeters and thus provide the team with critical physiologic data to allow better and more timely decision making and intervention.

\section{Pro/cons of the Lifestart ${ }^{\mathrm{Tm}}$ Trolley}

Pro: The Lifestart Trolley is the only commercial available device for clinical use (FDA-approved, Class 1) in the United States and Europe (CE marking). The greatest benefits are its portability, size, and inclusion of needed equipment, which can be used in an operative environment. This was the first device that has been tested and used in a number of multicenter and international trials. Such 
Fig. 2 Nooma cart. Labeled computer generated mockup (left panel) and cart use during a cesarean section (right panel).

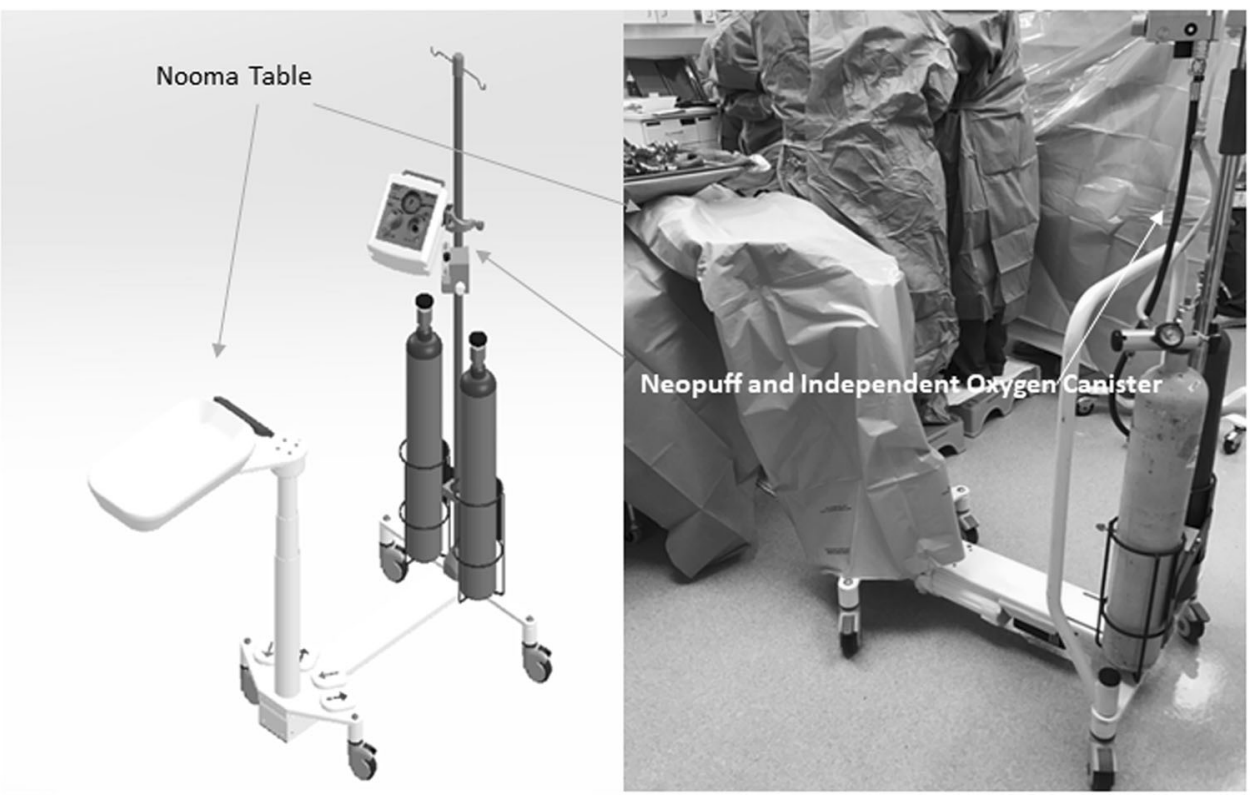

experience has allowed clinicians to provide input and refinement of the trolley, which has now been available for over a decade.

Cons: In clinical trials, the lack of transmitted heat with the bed was an issue as it could only be increased to $38^{\circ} \mathrm{C}$ (now $39^{\circ} \mathrm{C}$ in the new version). To overcome this, a chemical mattress was placed on top of the platform to provide heat for the first minute of life. The logistical issues of providing resuscitation on the cord have been previously described [20] in a previous paper, which details the limited space of providers and access to equipment. This experience may not be generalizable to other institutions or countries because of major institutional differences and study limitations. Vaginal deliveries in the United States are performed with delivery beds consistently being broken down and women placed in lithotomy position. This maternal positioning creates enough space for the LifeStart Trolley to only be placed next to the vagina. In the United Kingdom, vaginal deliveries occur without the beds being broken down allowing for the infant to be placed next to the mother. In their experience, providers rated the resuscitation trolley as the same, better, or much better than conventional equipment in $86 \%$ of their cases [13]. These issues must be improved upon with evidence for its use in larger randomized controlled trials before the LifeStart Trolley can be utilized as standard of care in the United States. Katheria et al. made a number of modifications to this trolley for local use, which include the attachment point for a small video camera, and the addition of a pulse oximeter. In addition, they added software that acquires the digital signals from the camera and oximeter. Additional devices may be able to store these in a readable chart like display along with the simultaneous video recording allowing critical review of the resuscitation. Currently, there are two other trials (NCT02742454, NCT02671305) that are using the Lifestart Trolley in Europe and the United States [21, 22].

\section{NOOMA cart (Maternal Life, LLC, USA)}

Lee et al. developed a device for cord management occurred in the setting of the Safety Learning Laboratory for Neonatal and Maternal Care, which began with funding from the Agency for Healthcare Research \& Quality (Fig. 2). In their lab, the goal was to be multidisciplinary in two ways: (1) connecting the work of all of the clinicians in labor and delivery, including obstetricians, pediatricians, anesthesiologists, and nurses; and (2) to gain insights from nonclinical perspectives, such as engineering, industrial design, and human factors. Cord management was identified as a clinical scenario where care was evolving and would benefit from a multidisciplinary inquiry. A data collection across California NICUs had shown that even after the recommendations for DCC had been implemented, many newborns were continuing to receive immediate cord clamping [23]. This was in the context of how to improve cord management in both situations where neonatal resuscitation is not needed, as well as to prepare for the potential that clinical trials may lead to broadening the recommendation for DCC to newborns that require resuscitation.

The lab utilized a design thinking framework, and involved a series of simulated vaginal and cesarean deliveries with obstetric and pediatric providers, simulating childbirth, DCC, and resuscitation with cord intact [24]. Simulations of vaginal and cesarean deliveries in our perinatal simulation lab, which involved recruiting pediatric and 
obstetric teams were videoed and debriefed. The clinical scenarios were deliveries of term and preterm infants that did or did not appear to require positive pressure ventilation at birth, with implementation of DCC. The scenarios concluded with the movement of the infant from the mother to the radiant warmer using the prototype cart. The debriefings focused on challenges to conducting the clinical scenario optimally. Broad areas to address in developing prototypes were maintaining sterility, equipment (such as a flat surface for the baby), mobility for infant movement when needed, space and workflow to account for multiple providers in a small space, and the need for clear communication. A key limiting aspect of a potential solution was the length of umbilical cord, and therefore, prototyping solutions were intended to maximize the use of a device to account for relatively shorter umbilical cords that would occur more frequently in preterm infants.

With that framework, the NOOMA cart was designed to have a bed space that had the ability to move up and down, as well as telescope in and out in order to get closer to the infant. The bed portion of the cart can maneuver into a space between a pediatric provider and obstetric provider during cesarean delivery. The cart holds gas canisters and is designed to facilitate the use of a T-piece resuscitator. It is also designed to allow for transport of the infant from the mother after the cord is clamped to the radiant warmer (Fig. 2).

\section{Clinical studies to date}

There are no clinical trials to date. Currently, the device is being tested under an approved protocol at Lucile Packard Children's Hospital Stanford for feasibility and prototype improvement. The initial phase involves deliveries of lowrisk term infants without requirement for resuscitation with informed consent from mothers and healthcare providers. This phase will test the ability of the cart to facilitate cord management in regard to ergonomics, safety, and with the goal to obtain qualitative feedback and potentially refine the prototype. The device has been used on 20 deliveries of term infants with plans to use it further on similar number of preterm infants.

\section{Pro/cons of the Nooma cart}

The NOOMA cart was designed to overcome some issues that were seen with other devices. For example, a pivoting table that telescopes and extends from the stand and can swerve side to side can overcome issues with cord length. The smaller table allows additional personnel to be able to squeeze into the sterile field, albeit still a confined space. Temperature was addressed by allowing a chemical warming mattress to be placed underneath the sterile drape that covers the tray.
The device has not been tested as part of any clinical trials as of yet to evaluate the potential improvements in temperature, spacing, and clinical use. Monitoring equipment is not visible to the clinician leading the resuscitation (currently is situated behind them). Additional setup for heart rate and oxygen monitoring is not currently part of the bed setup. The table currently moves up and down with the use of foot pedals, which may be challenging in the midst of a resuscitation; a newer model is in development to improve single-user control of elevation and retraction of the table.

\section{INSPiRe Trolley (Integrated Neonatal Support on Placental Circulation with Resuscitation, Alberta Health Services, Canada)}

In collaboration with biomedical engineers with Alberta Health Services, Canada in 2014, researchers in Calgary developed a resuscitation trolley to be used to initiate resuscitative care for newborns while on placental circulation. The trolley-Integrated Neonatal Support on Placental Circulation with Resuscitation (INSPiRe) was a heightadjustable resuscitation platform mounted with standard newborn resuscitative equipment and was independent of external power or gas supplies. Development of this batterypowered mobile, medical grade steel resuscitation platform equipped with a scale, pre-warmed gel mattress, oxygen and air tanks, blender, T-piece resuscitator, pulse oximeter and suction device was funded by the section of neonatology.

The device improved versatility in accessing crowded delivery room spaces and allowed the patient to be transferred to the warmer for ongoing care while receiving continuous respiratory care in transit. The ability of the trolley to be height-adjustable, allowed for vaginal deliveries where the bed was broken or unbroken. In the latter, the proximity of the resuscitative equipment and gases on the trolley, allowed for resuscitation of the infant on the maternal bed. The unbroken bed is the preferred delivery mode in vaginal deliveries with the smaller gestational ages in our institution.

The easily maneuverable platform without the need for external power and gas support, minimized trip hazards during the intervention. Training of personnel involved in using the resuscitation equipment and support from obstetrics providers occurred in tandem in the last year of the development of the INSPiRE Version 1 Trolley (Fig. 3, Panel A). The device received Canadian Standards Association approval and approval from Health Canada as Class 1 device with restricted approval for single institution research-related use. 


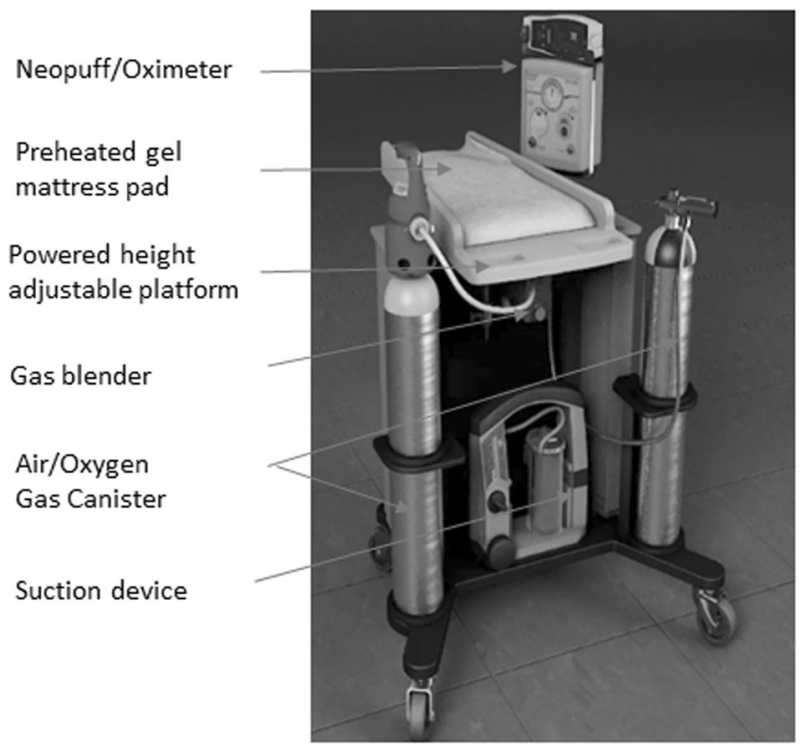

Fig. 3 Inspire Bed. Sketch of device with addtional elements labeled.

\section{Clinical studies to date}

A prospective pilot feasibility study was conducted on 20 preterm infants with gestational age between $260 / 7$ and 36 6/7 weeks delivered vaginally at Foothills Medical Centre in Calgary [25]. Fetuses with suspected pre-existing volume overload, estimated fetal weight less 3rd centile, triplets or higher order multiples and monochorionic multiples were excluded from the study.

Resuscitative care following Neonatal Resuscitation Program guidelines included assessment, stimulation, suction, and placement of pre-ductal saturation probe by $30 \mathrm{~s}$. Further assessment was performed at 30, 60, and $90 \mathrm{~s}$ intervals. The umbilical cord was clamped at $90 \mathrm{~s}$, and baby was transported to the warmer on the INSPiRe Trolley while continuing to receive resuscitative care. Twenty infants born (Median (range) gestational age was 31 (28-36) weeks and birth weight was 1655 (1270-2650) g) vaginally were managed using INSPiRe.

Several issues were identified in the first model. The weighing scale was found to be cumbersome and unreliable. The exterior was open to splash contamination, and required a plastic cover with use, to manage the cleaning process to disinfect between uses.

A second version was developed in 2017 (Fig. 3). This device was developed using a medical grade ceramic surface mounted on a steel enclosed base. This esthetically improved device was designed to reduce the footprint of the previous version, minimize splash contamination of internal systems, and improve disinfection and cleaning, and facilitate the ability to increase the variable height reach to access babies being born by cesarean section. The weighing scale was removed. It also utilized a stronger uninterruptible power source to drive the lift mechanism and mounted devices, including a humidification system to offer initial resuscitation using heated humidified gases for extremely preterm infants.

This trolley has been used successfully as part of the ongoing VentFirst Trial (NCT02742454) including infants born at 23 weeks 0 days through 28 weeks 6 days randomized to receive CPAP or positive pressure ventilation from 30 to $120 \mathrm{~s}$. To date, there have been 51 preterm infants randomized to receive the intervention during resuscitation utilizing INSPiRE version 2, of them, 22 were $<27$ weeks gestation. Procedures and training are in place to facilitate twin deliveries.

\section{Pro/cons of the INSPirRE Trolley}

The trolley has unrestricted maneuverability due to its compact size and independent gas and power support. The platform has extended height reach, designed to offer close access to operating tables for cesarean births as well as vaginal deliveries. The bed delivers heated humidified gases for resuscitation, mounted with equipment to support care during transition. There is reduced splash risk to equipment to facilitate cleaning after use. Pilot data have been reported including temperature and it has been trialed in preterm infants.

The trolley is not commercially available, and there is limited space to mount multifunction monitors to enhance research capability. There is limited use on extremely preterm infants (23-26 weeks). A 430-g infant had a short cord at delivery by caesarian section and the infant could not be placed on the platform. This infant was resuscitated on drapes just below the incision.

\section{Concord Birth Trolley (Concord Neonatal, Netherlands)}

At Leiden University Medical Center (LUMC) in the Netherlands, the development of a new purpose-built resuscitation table was started in 2013 as a collaborative initiative of the Neonatal, Obstetrical, and Medical Engineering Departments. The Concord Birth Trolley (Fig. 4) was designed to provide full standard care in stabilization of (preterm) infants at birth with intact umbilical cord. The length of the umbilical cord was recognized as an important limiting factor in a large proportion of infants. For the design the following preconditions were used: (1) complete standard care in stabilization of the infant can be provided, (2) the vital parameters of the infant can be monitored, (3) there is a possibility to stabilize the infant even when the cord is very short, (4) there is no interference of the working field of the neonatal care provider with the obstetrical care for the mother. 
Fig. 4 Concord Birth Trolley. Labeled prototype (left panel). Sketch of trolley during delivery (right panel).

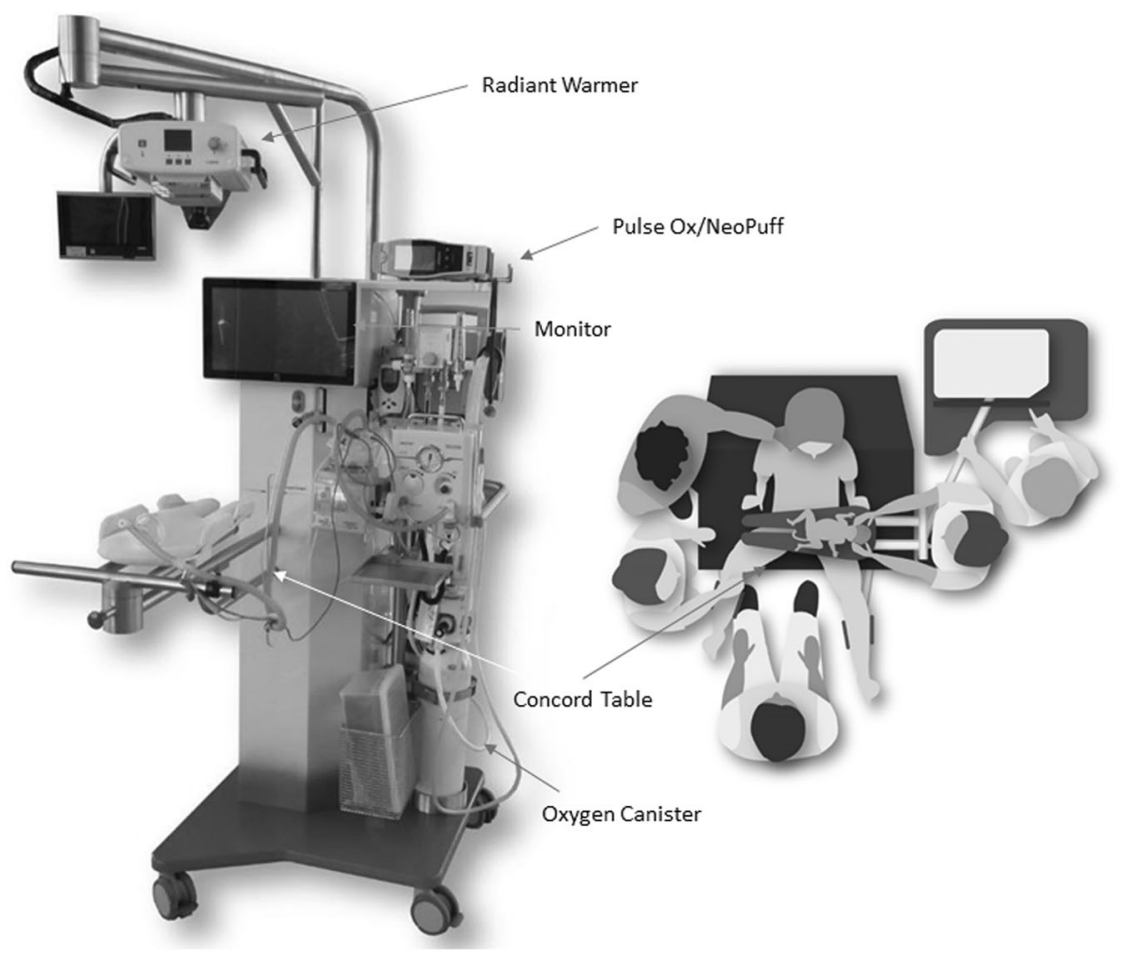

The design process has resulted in successive versions of the Concord. One of the unique features of the Concord Trolley is the platform design, which has a slit for the umbilical cord. The platform is height-adjustable and can be turned and placed just above the pelvis of the mother. This allows the infant to be kept close to the mother without stretching the umbilical cord. The equipment needed for stabilization and resuscitation can be incorporated in the table, including a radiant heater, T-piece device, pulse oximeter, oxygen blender, and suctioning equipment. Ample space for additional instruments such as a respiratory function monitor or a cerebral oximeter. There is space available on the Concord for two gas tanks (Europe only). During a cesarean section, the platform of the Concord is easily draped with sterile wraps. The prototype of the Concord was designed at the LUMC and is now commercially available in the EU and Australia. A start-up company (Concord Neonatal) has been launched in 2017 for the commercialization of the Concord.

\section{Clinical studies to date}

So far, clinical studies using the Concord Birth Trolley have all aimed at PBCC, meaning that stabilization of the infant is performed with intact umbilical cord and that the cord is only clamped when the infant is considered respiratory "stable" (heart rate $>100, \mathrm{SpO}_{2}>90 \%, \mathrm{FiO}_{2}<0.4$ ). As a result, timing of cord clamping varies and is not time-based, but individually determined by the transitional status of the infant. A recently completed feasibility study $(\mathrm{ABC} 1, N=$
37) in preterm infants demonstrated that PBCC using the Concord was feasible and safe [26]. The observations showed less bradycardia and hypoxia at birth compared to historical data, supporting the more stable transition. The average cord clamping time was more than $4 \mathrm{~min}$, which may allow the preterm infants to benefit from a more optimal placental transfusion.

In the $\mathrm{ABC} 2$ trial, preterm infants (gestational age $<32$ weeks) were randomized to either standard DCC (30-60 s) or PBCC using the Concord [27]. Thirty-seven infants were included, showing that stabilization by performing PBCC and using the Concord was effective and resulted in a mean cord clamping time of 5:49 min. Feasibility and safety for mother and infant were confirmed. In order to assess the clinical benefit of the procedure and the Concord, in January 2019 a large randomized clinical trial (ABC3, NCT03808051) aiming for 660 preterm infants (gestational age $<30$ weeks) was initiated in the Netherlands, comparing important clinical outcomes between standard DCC and PBCC. In addition, the Concord is being used in the PinC trial (NCT04373902), comparing PBCC to ICC in newborn infants with a congenital diaphragmatic hernia. Results of these two large ongoing trials are to be awaited.

\section{Pro/cons of the Concord Birth Trolley}

The Concord Birth Trolley is a fully equipped resuscitation table with specifically designed platform for the infant, including an incorporated slit for the umbilical cord. 


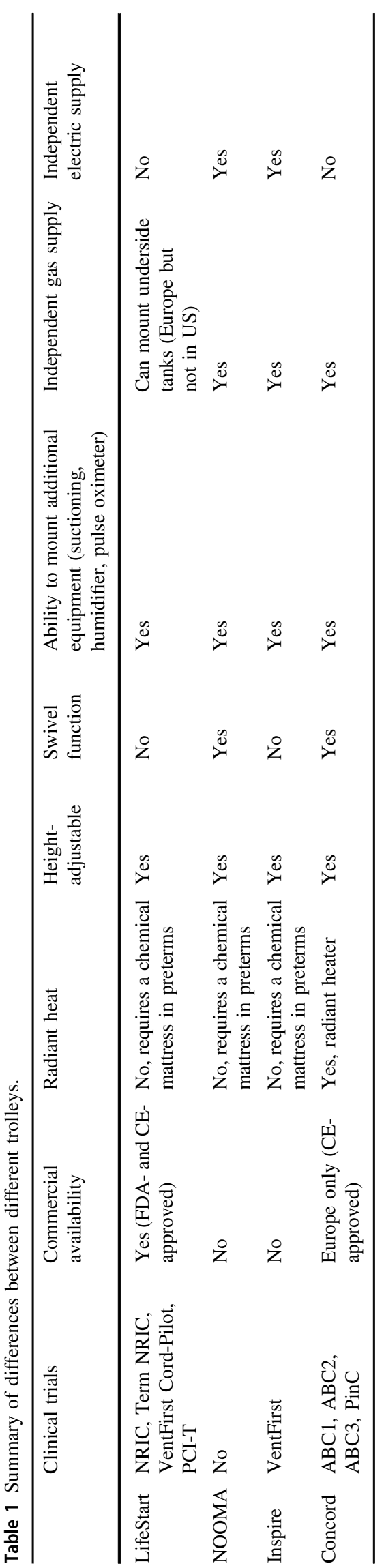

Optimal placement is possible due to the height-adjustable and rotating platform, without interfering with the obstetrical workspace. The Concord Birth Trolley holds ample space for standard and additional equipment and devices, including gas tanks. The table is certified for clinical use and commercially available in Europe and Australia but not in the United States.

When supplied with all equipment and gas tanks, the Concord Birth Trolley is quite heavy and robust, making maneuvering the table in very small crowded spaces more challenging. The Concord Birth Trolley is not certified for transportation, requiring a transfer of the infant to a transport incubator after stabilization.

\section{Discussion}

The physiological benefits of DCC with concomitant establishment of breathing have been established in animal and human studies. Ongoing trials are currently focused on the benefits of establishing cardiorespiratory stability in premature infants born with reduced respiratory drive and pulmonary immaturity while attached to the placental circulation. By providing a placental transfusion to the sickest infants, we may see a reduction in morbidities such as the incidence of IVHs and decreased requirement for postnatal volume expansion, use of inotropes and the need for blood transfusions.

The devices feature common elements, which offer the ability to access the delivering fetus, using a platform mounted with standard neonatal resuscitation equipment to start resuscitative interventions from birth prior to cutting the umbilical cord (Table 1). The desirable features for such devices include unrestricted maneuverability within busy delivery suites, height-adjustable and swivel-able platform to facilitate access to the fetus being born vaginally and by caesarian section, a heat source to maintain thermoregulation, the use of heated and humidified gases for resuscitation of very premature infants, and the ability to monitor the newborn from birth deploying multichannel physiological monitors. These features are aimed at offering resuscitative care using contemporary standards, by monitoring the newborn while on placental circulation. Additional monitoring such as pulse oximetry, a pneumotach/hot wire with respiratory parameters displayed, oxygen analyzer with digital output, a video camera, and a real-time temp probe would provide additional information to improve resuscitation both during and provide feedback to the team for quality assurance and research. They also provide additional oversight in a less resourced setting (i.e., fewer nurses and respiratory personnel that have access to the infant). The use of these devices during a resuscitation with an intact cord may improve the resuscitative efforts by improving heart 
rate, arterial oxygenation, and lung aeration, but until better monitoring is in place this will not be clear.

There are still limitations to the resuscitation trolleys. Future areas should focus on provision of targeted specific respiratory support with minimal handling and uninterrupted respiratory support providing a seamless transition from the delivery room to care in the neonatal intensive care unit (i.e., a transport bed). How and when these devices should be used is also unclear (i.e., high-risk deliveries vs. all deliveries).

\section{Conclusion}

Resuscitation on placental circulation for babies who require respiratory support from birth is currently under investigation and not yet recommended for routine use. Devices described in this review have common and desirable features, which may be further developed. Currently, these devices are used for quality improvement and research and not for all high-risk deliveries. Ongoing clinical trials and testing of such devices and approaches will inform providers on potential clinical benefits for these infants.

Acknowledgements This project was supported by Grant Number P30HS023506 from the Agency for Healthcare Research and Quality. The content is solely the responsibility of the authors and does not necessarily represent the official views of the Agency for Healthcare Research and Quality. The authors thank Dr Neil Finer for his edits and review of the manuscript.

\section{Compliance with ethical standards}

Conflict of interest The authors declare no competing interests.

Publisher's note Springer Nature remains neutral with regard to jurisdictional claims in published maps and institutional affiliations.

\section{References}

1. Wyckoff MH, Aziz K, Escobedo MB, Kapadia VS, Kattwinkel J, Perlman JM, et al. Part 13: neonatal resuscitation: 2015 American Heart Association Guidelines update for cardiopulmonary resuscitation and emergency cardiovascular care (Reprint). Pediatrics. 2015;136:S196-218.

2. Textbook of neonatal resuscitation (NRP). Weiner GM, Zaichkin $\mathbf{J}$ editors. 7th ed. Elk Grove Village, IL, 2016.

3. Mercer JS, Erickson-Owens DA, Vohr BR, Tucker RJ, Parker AB, $\mathrm{Oh} \mathrm{W}$, et al. Effects of placental transfusion on neonatal and 18 month outcomes in preterm infants: a Randomized Controlled Trial. J Pediatr. 2016;168:50-55.e1.

4. Andersson O, Lindquist B, Lindgren M, Stjernqvist K, Domellof M, Hellstrom-Westas L. Effect of delayed cord clamping on neurodevelopment at 4 years of age: a Randomized Clinical Trial. JAMA Pediatr. 2015;169:631-8.
5. Glass HC, Costarino AT, Stayer SA, Brett CM, Cladis F, Davis PJ. Outcomes for extremely premature infants. Anesth Analg. 2015; 120:1337-51.

6. Redmond A, Isana S, Ingall D. Relation of onset of respiration to placental transfusion. Lancet. 1965;1:283-5.

7. Philip AG, Yee AB, Rosy M, Surti N, Tsamtsouris A, Ingall D. Placental transfusion as an intrauterine phenomenon in deliveries complicated by foetal distress. Br Med J. 1969;2:11-3.

8. Vyas H, Milner AD, Hopkins IE. Intrathoracic pressure and volume changes during the spontaneous onset of respiration in babies born by cesarean section and by vaginal delivery. J Pediatr. 1981;99:787-91.

9. Katheria AC, Lakshminrusimha S, Rabe H, McAdams R, Mercer JS. Placental transfusion: a review. J Perinatol. 2017;37:105-11.

10. Ersdal HL, Linde J, Mduma E, Auestad B, Perlman J. Neonatal outcome following cord clamping after onset of spontaneous respiration. Pediatrics. 2014;134:265-72.

11. Nevill E, Meyer MP. Effect of delayed cord clamping (DCC) on breathing and transition at birth in very preterm infants. Early Hum Dev. 2015;91:407-11.

12. Bhatt S, Alison BJ, Wallace EM, Crossley KJ, Gill AW, Kluckow $\mathrm{M}$, et al. Delaying cord clamping until ventilation onset improves cardiovascular function at birth in preterm lambs. J Physiol. 2013;591:2113-26.

13. Thomas MR, Yoxall CW, Weeks AD, Duley L. Providing newborn resuscitation at the mother's bedside: assessing the safety, usability and acceptability of a mobile trolley. BMC Pediatr. 2014; 14:135.

14. Duley L, Dorling J, Pushpa-Rajah A, Oddie SJ, Yoxall CW, Schoonakker B, et al. Randomised trial of cord clamping and initial stabilisation at very preterm birth. Arch Dis Child Fetal Neonatal Ed. 2017;103:F6-F14.

15. Katheria A, Poeltler D, Durham J, Steen J, Rich W, Arnell K, et al. Neonatal resuscitation with an intact cord: a Randomized Clinical Trial. J Pediatr. 2016;178:75-80.e3.

16. Katheria AC, Brown MK, Faksh A, Hassen KO, Rich W, Lazarus $\mathrm{D}$, et al. Delayed cord clamping in newborns born at term at risk for resuscitation: a feasibility Randomized Clinical Trial. J Pediatr. 2017;187:313-7.e1.

17. Sæther E, Gülpen FR, Jensen C, Myklebust T, Eriksen BH. Neonatal transitional support with intact umbilical cord in assisted vaginal deliveries: a quality-improvement cohort study. BMC Pregnancy Childbirth. 2020;20:496.

18. Foglia EE, Ades A, Hedrick HL, Rintoul N, Munson DA, Moldenhauer $\mathrm{J}$, et al. Initiating resuscitation before umbilical cord clamping in infants with congenital diaphragmatic hernia: a pilot feasibility trial. Arch Dis Child Fetal Neonatal Ed. 2020;105:322-6.

19. Hoyle ES, Hirani S, Ogden S, Deeming J, Yoxall CW. Quality improvement programme to increase the rate of deferred cord clamping at preterm birth using the Lifestart trolley. Arch Dis Child Fetal Neonatal Ed. 2020;105:652-5.

20. Katheria AC, Sorkhi SR, Hassen K, Faksh A, Ghorishi Z, Poeltler D. Acceptability of bedside resuscitation with intact umbilical cord to clinicians and patients' families in the United States. Front Pediatr. 2018;6:100.

21. Winter J, Kattwinkel J, Chisholm C, Blackman A, Wilson S, Fairchild K. Ventilation of preterm infants during delayed cord clamping (VentFirst): a pilot study of feasibility and safety. Am J Perinatol. 2017;34:111-6.

22. Pratesi S, Montano S, Ghirardello S, Mosca F, Boni L, Tofani L, et al. Placental Circulation Intact Trial (PCI-T)-resuscitation with the placental circulation intact vs. cord milking for very preterm infants: a feasibility study. Front Pediatr. 2018;6:364. 
23. Tran CL, Parucha JM, Jegatheesan P, Lee HC. Delayed cord clamping and umbilical cord milking among infants in california neonatal intensive care units. Am J Perinatol. 2020;37:151-7.

24. Lapcharoensap W, Cong A, Sherman J, Schwandt D, Crowe S, Daniels K, et al. Safety and ergonomic challenges of ventilating a premature infant during delayed cord clamping. Children. 2019;6:59.

25. Irvine LMA, Kowal D, Soraisham A, Cooper S, Stritzke A, Rabi $\mathrm{Y}$, et al. Integrated neonatal support on placental circulation with resuscitation (INSPiRe): a feasibility study. Proceedings of the 10th American Pediatrics Healthcare \& Pediatric Infectious Diseases Congress. Toronto, Canada; 2017.

26. Brouwer E, Knol R, Vernooij ASN, van den Akker T, Vlasman PE, Klumper FJCM, et al. Physiological-based cord clamping in preterm infants using a new purpose-built resuscitation table: a feasibility study. Arch Dis Child Fetal Neonatal Ed. 2019;104:F396-402.

27. Knol R, Brouwer E, van den Akker T, DeKoninck P, van Geloven N, Polglase GR, et al. Physiological-based cord clamping in very preterm infants-randomised controlled trial on effectiveness of stabilisation. Resuscitation. 2020;147:26-33. 